\title{
On Enabling Compliance of Cross-organizational Business Processes
}

\author{
David Knuplesch ${ }^{1}$, Manfred Reichert ${ }^{1}$, Walid Fdhila ${ }^{2}$, and \\ Stefanie Rinderle-Ma ${ }^{2}$ \\ ${ }^{1}$ Institute of Databases and Information Systems, Ulm University, Germany \\ 2 Faculty of Computer Science, University of Vienna, Austria \\ \{david.knuplesch,manfred.reichert\}@uni-ulm.de, \\ $\{$ walid.fdhila, stefanie.rinderle-ma\}@univie.ac.at
}

\begin{abstract}
Process compliance deals with the ability of a company to ensure that its business processes comply with domain-specific regulations and rules. So far, compliance issues have been mainly addressed for intra-organizational business processes, whereas there exists only little work dealing with compliance in the context of cross-organizational processes that involve multiple business partners. As opposed to intraorganizational processes, for a cross-organizational process, compliance must be addressed at different modeling levels, ranging from interaction models to public process models to private processes of the partners. Accordingly, there exist different levels for modeling compliance rules. In particular, we distinguish between local compliance rules of a particular partner and global compliance rules to be obeyed by all partners involved in the cross-organizational process. This paper focuses on checking the compliance of interaction models. For this purpose, we introduce the notion of compliability, which shall guarantee that an interaction model is not conflicting with a set of imposed global compliance rules.
\end{abstract}

\section{Introduction}

Business process compliance has been identified as one of the core challenges for process-aware information systems [23]. So far, the focus has been on intraorganizational business processes, and a variety of proposals for checking the compliance of a business process with domain-specific regulations and rules in different phases of the process life cycle have been made [1,20,13, 21,22]. Besides few approaches (e.g., business contracts $[2,11]$ ), compliance checking for crossorganizational processes has been neglected so far, even though being crucial in collaborative settings $[14,16]$. Therefore, the consideration of compliance rules in the context of cross-organizational processes and the provision of techniques for checking them are indispensable. Compared to approaches checking the compliance of intra-organizational business processes, however, additional challenges

\footnotetext{
* This work was done within the research project $\mathrm{C}^{3}$ Pro funded by the German Research Foundation (DFG) under project number RE 1402/2-1, and the Austrian Science Fund (FWF) under project number I743.
} 
emerge [14]. In particular, process compliance must be ensured at different levels. Furthermore, compliance checking must cope with the fact that parts of crossorganizational processes are not known by all partners, e.g. due to privacy reasons.

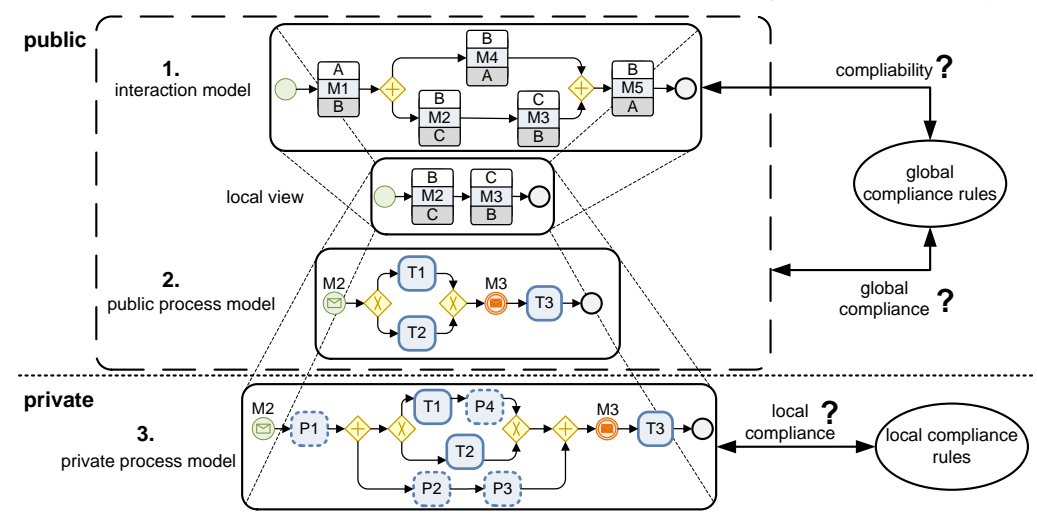

Fig. 1. Different levels of compliance rules in a cross-organizational setting

Consider Fig. 1: Compliance rules relevant for setting up and running crossorganizational processes refer to different levels as known from interaction modeling [5]. First, the global view of the interactions (i.e. messages exchanged) between the partners of a cross-organizational process (i.e., the interaction model) provides the top level. Second, each partner defines a public model of its process taking its local view on the interaction model into account. Accordingly, a local view refers to the behavior of the interaction model from the viewpoint of a particular partner. Note that public models must conform with the behavior of the respective local view. Finally, each partner maintains its own private process model, which not only comprises activities for exchanging messages with the other partners, but also private activities not relevant for the interactions with the partners; i.e., due to privacy, a partner usually does not reveal all details about its private processes to the other partners. However, the private processes must conform with the public process model. Semantic constraints in respect to such private processes are denoted as local compliance rules. In turn, global compliance rules are imposed on interaction models and public process models. Altogether, the public parts of a cross-organizational process include the interaction model, public process models, and global compliance rules. In turn, private process models and local compliance rules constitute private parts.

As opposed to intra-organizational processes, in the context of cross-organizational processes we must consider three levels of compliance. First, we must deal with local compliance rules that constrain private partner processes. Second, we must support global compliance rules that constrain the public parts of a cross-organizational process scenario. Third, interaction models must enable the partners to model both public and private processes meeting the global compliance rules. This requires interaction models being not in conflict with the set of global compliance rules.

This paper focuses on the latter level of compliance and provides a novel 
correctness criterion, which we denote as compliability in the following. Compliability refers to the ability of an interaction model to comply with a given set of global compliance rules without knowing all details of the private and public process models of the partners. We denote an interaction model as not compliabile, if it conflicts with the given set of global compliance rules. In particular, we present an approach enabling automated compliability checking of interaction models against a given set of imposed global compliance rules. Note that compliability must be ensured before the partners specify their public and private process models. Therefore, our approach extends interaction models with additional control flow structures and activities to approximate the not yet specified behavior of partners. Furthermore, it merges and adapts the global compliance rules, before checking compliability through the application of model checking techniques.

Note that this paper focuses on compliability checking of interaction models at build time, but does not consider any other phase of the process life cycle (e.g., execution and change time). Furthermore, we focus on compliance rules related to control-flow and do not explicitly address data, resources, or time. Finally, our approach requires from the partners to publish the set of activities they use for specifying their public and private processes.

The remainder of this paper is structured as follows: Sect. 2 illustrates an example from the healthcare domain. Sect. 3 provides algorithms for compliability checking as the main contribution of this paper. Sect. 4 discusses related work and Sect. 5 closes with a discussion and outlook.

\section{Example}

Figs. 2 and 3 depict an example of a cross-organizational process from the healthcare domain using the BPMN 2.0 standard. This process involves three partners: gynecologist, laboratory, and hospital. In particular, Fig. 2 depicts the interactions (i.e., messages exchanged) between these partners. In turn, Fig. 3 shows their public process models. Note that the public process model of the hospital is simplified due to space limitations. Tab. 1 shows and classifies examples of compliance rules imposed on the cross-organizational process from Figs. 2 and 3.

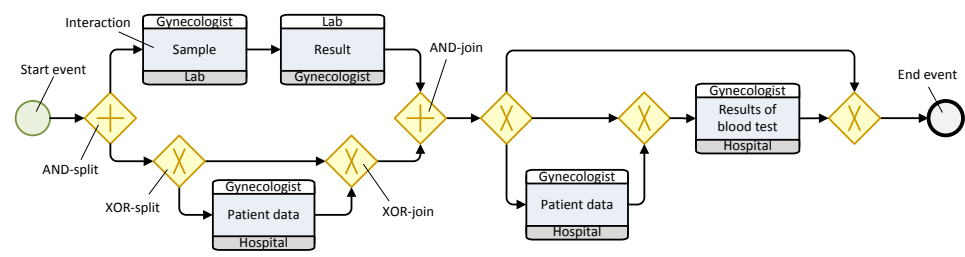

Fig. 2. Interaction model of a healthcare scenario [14]

The interaction model from Fig. 2 is compliable with the set of global compliance rules, which comprise $r_{1}$ and $r_{2}$. This can be easily shown, since the collaboration of the public processes (cf. Fig. 3) complies with $r_{1}$ and $r_{2}$. In general, however, public processes will not have been specified yet when verifying compliability (cf. Fig. 1). 


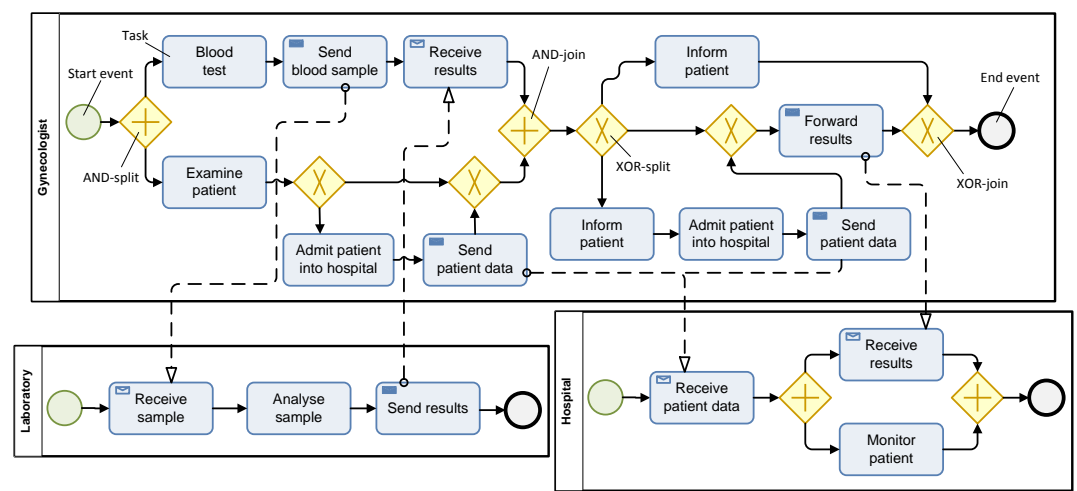

Fig. 3. Public models of a healthcare scenario [14]

\begin{tabular}{|c|l|l|}
\hline- & Classification & Compliance rule \\
\hline$r_{1}$ & Global compliance Rule & After a blood test, the blood sample has to be analyzed. \\
\hline$r_{2}$ & Global compliance rule & After the patient is admitted to the hospital, she must be monitored. \\
\hline$r_{3}$ & $\begin{array}{l}\text { Local compliance rule of } \\
\text { gynecologist }\end{array}$ & The patient must be informed about the results of a blood test. \\
\hline
\end{tabular}

Table 1. Examples of compliance rules

\section{Compliability Checking}

When setting up a cross-organizational process, compliability constitutes a semantic correctness criterion to be considered when designing interaction models. It ensures that interaction models do not conflict with the set of imposed global compliance rules. Consequently, if an interaction model is not compliable, the involved partners are unable to implement their public and private processes in such a way that the overall cross-organizational process satisfies all imposed compliance rules. As input our approach takes the interaction model $\mathcal{I}$, the set of global compliance rules $G R$ expressed in terms of linear temporal logic (LTL), the set of partners $\mathcal{P}$, and for each partner $p \in \mathcal{P}$ the set of activities $A_{p}$ that $p$ may execute.

Similar to approaches checking compliance of intra-organizational processes, we apply model checking to ensure compliability. As opposed to these intraorganizational approaches, however, we do not want to show that all possible executions of a model comply with all compliance rules in $G R$, but that there exists at least one execution satisfying all compliance rules in $G R$, and hence $\mathcal{I}$ and $G R$ do not conflict. Furthermore, we cannot directly take the interaction model $\mathcal{I}$ as input for model checking, but must consider all tasks that might be additionally executed by partners. Thus, we cannot apply model checking directly, but have to add preprocessing steps.

Consider Fig. 4: We utilize the knowledge about the set of activities $A_{p}$ to enrich interaction model $\mathcal{G}$ with parts simulating the behavior of the partners involved (cf. Alg. 1), and obtain an extended interaction model (EIM) as result (cf. Fig. 5).This enrichment is expressed through the following constructs: 
sequence (SEQ), parallelism (PAR), choice (CHC), and repeated loop (RPT). Note that this does not require the interaction model to be well-structured.

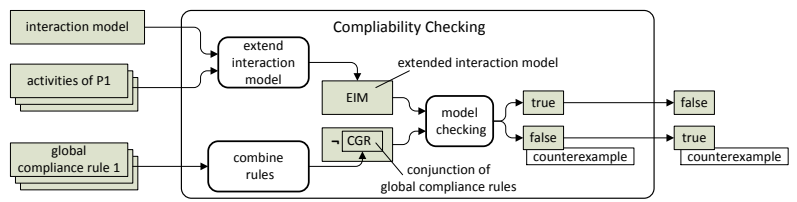

Fig. 4. Process of compliability checking
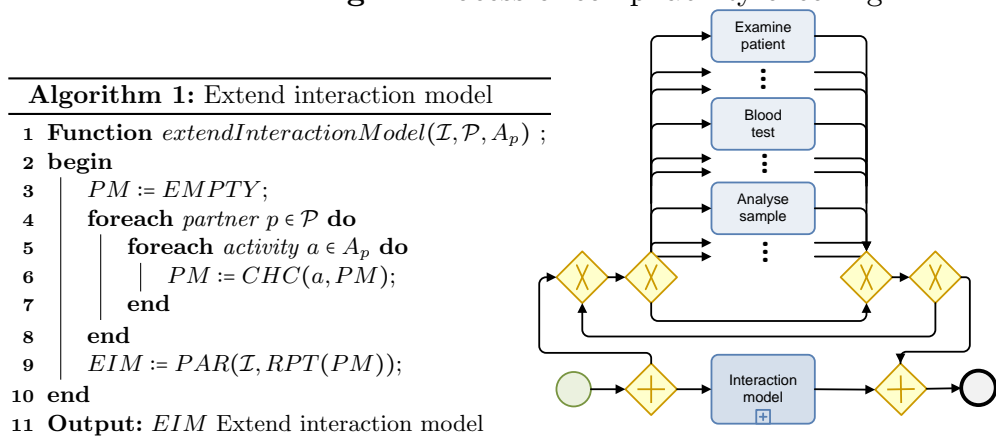

Fig. 5. Extended interaction model EIM

Next, we construct the conjunction of all global compliance rules $G C R$ in Alg. 2. Finally, we apply LTL model checking to the extended interaction model $E I M$ and the negation of $G C R$ in Alg. 3. In case the enriched model EIM is compliable, at least one trace $\tau$ is producible through EIM satisfying all global compliance rules. Consequently, the negated conjunction of the global compliance rules does not hold. For this case, explicit LTL model checking returns false (and outputs $\tau$ as counter-example), which means that compliabily holds. Otherwise, all traces violate at least one of the global compliance rules, and $E I M$ satisfies the negated formula. In this case, model checking returns true, but compliability is violated. Basically, our algorithm for compliability checking applies model checking over the enriched interaction model EIM and the negated conjunction of the global compliance rules, and then negates the result (cf. Alg. 3 and Fig. 4).
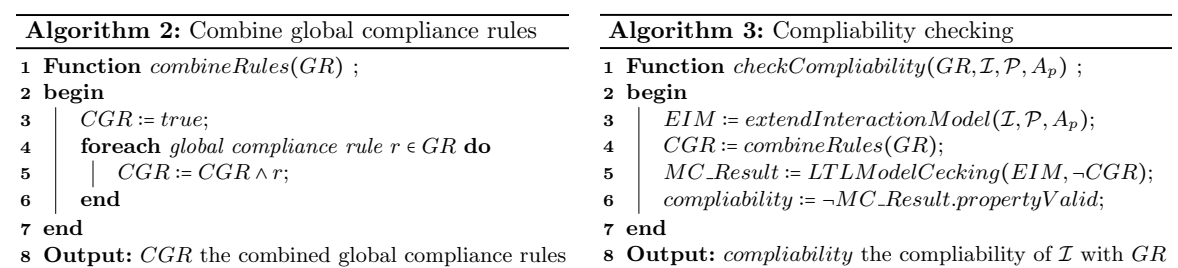

We have demonstrated the feasibility of our approach by a proof-of-concept prototype. We applied this prototype to different application scenarios including the sketched healthcare example [14]. More precisely, the presented compliance checking techniques have been implemented as plug-in of the Aristaflow BPM 


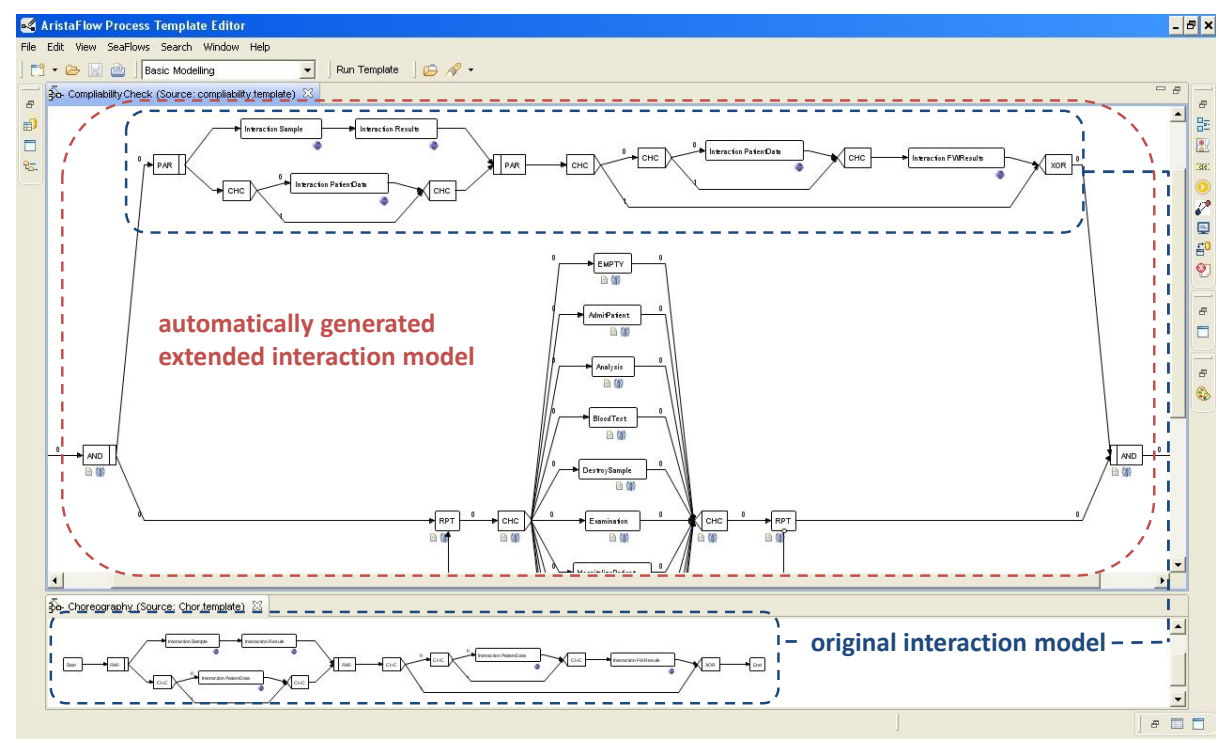

Fig. 6. Proof-of-concept prototype enabling compliability checking

Suite [3]. The upper part of Fig. 6 shows an automatically generated, extended interaction model for compliability checking, while the lower part depicts the original interaction model.

\section{Related Work}

In many domains, process execution is subject to compliance rules and restrictions that stem from laws, regulations, and guidelines (e.g. Basel or SarbanesOxley-Act) [23]. Existing approaches differ with respect to the process lifecycle phase in which compliance is considered [7]. Compliance rules are often considered as restrictions to the order in which process activities may be executed. In literature, there exist approaches formalizing these rules with temporal logic $[1,10]$, patterns $[6]$, or graphical notations $[1,18]$. To check whether compliance rules are fulfilled by a process model at build time, most approaches apply model checking $[1,10,19,12]$. Furthermore, business process compliance along the process lifecycle is discussed in [20,13].

Only little work exists, which deals with compliance of cross-organizational processes $[2,11]$. In particular, compliability of interaction models with a given set of compliance rules has not been addressed yet.

Various other issues related to the correctness of cross-organizational processes and complementing compliability have been addressed. For example, [4, 8 ] discuss whether private processes are compatible with the public ones. In turn, $[17,15]$ introduce the notion of realizability of interaction models, i.e., to check whether involved partners are able to model public and private processes 
compatible with a particular interaction model. [9] discusses changes and their propagation in cross-organizational scenarios.

\section{Discussion and Outlook}

To ensure compliance of business processes with existing guidelines, standards, and laws is crucial for both intra-organizational and cross-organizational settings. However, existing proposals have only dealt with intra-organizational processes so far [14]. This paper constitutes an important step towards enabling compliance of cross-organizational business processes at different levels. In particular, we introduced the notion of compliability, i.e., the general ability of an interaction model to not conflict with a given set of compliance rules independent from the concrete process models of the partners.

However, compliability does not guarantee that there exists a compliant realization of an interaction model; i.e., public and private process models that comply with the global compliance rules. For example, consider the interaction model from Fig. 7 and the global compliance rules $r_{4}$ and $r_{5}$ from Tab. 2. As indicated by the process log being the output of compliability checking (cf. Fig. 7), there exists no conflict between the model and $r_{4}$ and $r_{5}$. Nevertheless, the partners are not able to specify compliant private and public processes, because the laboratory is unable to determine, when the patient is notified. Thus, laboratory can not determine when activity analyse sample may be started without violating $r_{5}$. This is caused by a missing interaction. In the example, it is easy to enhance the interaction model with an additional interaction to enable the partners to specify compliant private and public processes (cf. Fig. 7). Generally, compliability remains a necessary, but not sufficient precondition for the ability of the partners to specify their public and private models in such a way that the overall cross-organizational process satisfies the set of imposed compliance rules.

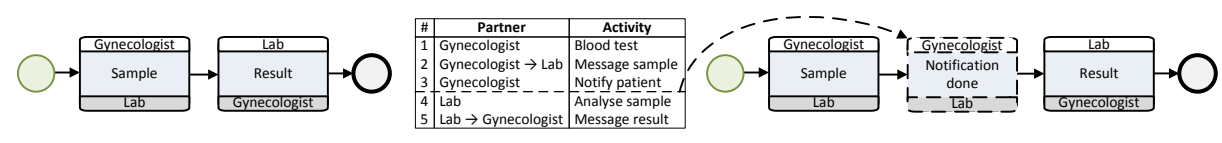

Fig. 7. An example indicating the limitations of compliability

\begin{tabular}{|c|l|l|}
\hline- & Classification & Compliance rule \\
\hline$r_{4}$ & Global compliance rule & After blood sample is sent to laboratory, the patient has to be notified. \\
\hline$r_{5}$ & Global compliance rule & $\begin{array}{l}\text { The analysis must start after the notification of the patient, but before } \\
\text { results are sent. }\end{array}$ \\
\hline
\end{tabular}

Table 2. Examples of compliance rules

In future work, we will present a comprehensive formal theory for compliance and related criteria (e.g., compliability) in cross-organizational processes. Further, we will present additional algorithms for checking compliability and global compliance as well as related semantic correctness criteria. Finally, we will consider additional process perspectives (e.g. data, time, resources) in the context of compliance and compliability checking. 


\section{References}

1. Awad, A., Decker, G., Weske, M.: Efficient compliance checking using BPMN-Q and temporal logic. In: BPM'08. pp. 326-341 (2008)

2. Berry, A., Milosevic, Z.: Extending choreography with business contract constraints. Int J Coop Inf Sys 14(2-3), 131-179 (2005)

3. Dadam, P., Reichert, M.: The ADEPT project: a decade of research and development for robust and flexible process support. Computer Science-Research and Development 23(2), 81-97 (2009)

4. Decker, G., Weske, M.: Behavioral consistency for B2B process integration. In: CAiSE'07. pp. 81-95 (2007)

5. Decker, G., Weske, M.: Interaction-centric modeling of process choreographies. Inf Sys 35(8) (2010)

6. Dwyer, M.B., Avrunin, G.S., Corbett, J.C.: Property specification patterns for finite-state verification. In: FMSP'98 (1998)

7. El Kharbili, M., et al.: Business process compliance checking: Current state and future challenges. In: MobIS'08. pp. 107-113 (2008)

8. Fdhila, W., Rouached, M., Godart, C.: Communications semantics for WS-BPEL processes. In: ICWS'08 (2008)

9. Fdhila, W., Rinderle-Ma, S., Reichert, M.: Change propagation in collaborative processes scenarios. In: CollaborateCom'12. pp. 452-461. IEEE Comp Press (2012)

10. Ghose, A.K., Koliadis, G.: Auditing business process compliance. In: ICSOC'07. pp. 169-180 (2007)

11. Governatori, G., Milosevic, Z., Sadiq, S.: Compliance checking between business processes and business contracts. In: EDOC'06. pp. 221-232 (2006)

12. Knuplesch, D., Ly, L.T., Rinderle-Ma, S., Pfeifer, H., Dadam, P.: On enabling data-aware compliance checking of business process models. In: ER'2010 (2010)

13. Knuplesch, D., Reichert, M.: Ensuring business process compliance along the process life cycle. Tech. Rep. 2011-06, University of Ulm (2011)

14. Knuplesch, D., et al.: Towards compliance of cross-organizational processes and their changes - research challenges and state of research. In: BPM Workshops. pp. 649-661 (2013)

15. Knuplesch, D., Pryss, R., Reichert, M.: Data-aware interaction in distributed and collaborative workflows: modeling, semantics, correctness. In: CollaborateCom'12. pp. 223-232. IEEE Comp Press (2012)

16. Leitner, M., Mangler, J., Rinderle-Ma, S.: Definition and enactment of instancespanning process constraints. In: WISE'2012. pp. 652-658 (2012)

17. Lohmann, N., Wolf, K.: Realizability is controllability. Web Services and Formal Methods pp. 110-127 (2010)

18. Ly, L.T., et al.: Design and verification of instantiable compliance rule graphs in process-aware information systems. In: CAiSE'10. pp. 9-23 (2010)

19. Ly, L.T., et al.: Seaflows toolset-compliance verification made easy for processaware information systems. Inf Syst Evolution pp. 76-91 (2011)

20. Ly, L.T., et al.: On enabling integrated process compliance with semantic constraints in process management systems. Inf Sys Frontiers 14(2), 195-219 (2012)

21. Maggi, F., et al.: Monitoring business constraints with linear temporal logic: an approach based on colored automata. In: BPM'11. pp. 132-147 (2011)

22. Ramezani, E., et al.: Separating compliance management and business process management. In: BPM Workshops. pp. 459-464 (2012)

23. Sadiq, S., Governatori, G., Naimiri, K.: Modeling control objectives for business process compliance. In: BPM'07. pp. 149-164 (2007) 